\title{
AUTOMATIC IN-SITU SELF-CALIBRATION OF A PANORAMIC TLS FROM A SINGLE STATION USING 2D KEYPOINTS
}

\author{
T. Medic ${ }^{1, *}$, H. Kuhlmann ${ }^{1}$, C. Holst ${ }^{1}$ \\ ${ }^{1}$ Institute of Geodesy and Geoinformation, Universität Bonn, Germany - (medic, kuhlmann, holst)@igg.uni-bonn.de
}

KEY WORDS: Self-Calibration, Laser Scanning, TLS, Point Cloud, Intensity Image, Feature Detector, Accuracy

\begin{abstract}
:
Terrestrial laser scanner (TLS) measurements are unavoidably affected by systematic influences due to internal misalignments. The magnitude of the resulting errors can exceed the magnitude of random errors significantly deteriorating the quality of the obtained point clouds. Hence, the task of calibrating TLSs is important for applications with high demands regarding accuracy. In recent years, multiple in-situ self-calibration approaches were derived allowing the successful estimation of up-to-date calibration parameters. These approaches rely either on using manually placed targets or on using man-made geometric objects found in surroundings. Herein, we widen the existing toolbox with an alternative approach for panoramic TLSs, for the cases where such prerequisites cannot be met. We build upon the existing target-based two-face calibration method by substituting targets with precisely localized 2D keypoints, i.e. local features, detected in panoramic intensity images using the Förstner operator. To overcome the detriment of the perspective change on the feature localization accuracy, we estimate the majority of the relevant calibration parameters from a single station. The approach is verified on real data obtained with the Leica ScanStation P20. The obtained results were tested against the affirmed target-based two-face self-calibration. Analysis proved that the estimated calibration parameters are directly comparable both in the terms of parameter precision and correlation. In the end, we employ an effective evaluation procedure for testing the impact of the calibration results on the point cloud quality.
\end{abstract}

\section{INTRODUCTION}

High-end panoramic TLSs are 3D measurement instruments with a wide field of view and point accuracy in the order of a few millimeters in the full working range. Combined with a fast acquisition rates and high measurement density, this assured their place as a standard tool for highly demanding engineering tasks such as deformation monitoring (Mukupa et al., 2017) and reverse engineering (Bianculli et al., 2016). Despite the tremendous efforts of the manufacturers, the TLSs are not geometrically perfect and their inner geometry can change over time due to wear and tear or suffered stress. The resulting misalignments cause systematic displacements of the measured points reducing the quality of the point cloud. In order to assure the high measurement accuracy, it is necessary to calibrate the instrument in certain time intervals to mathematically account for these systematic errors.

In the last two decades, multiple successful TLS calibration approaches have been proposed in the literature. Most of the existing approaches are in-situ approaches assuring up-to-date estimates of the calibration parameters (CPs) and relying on the environment found on a certain scene. They are mostly realized as self-calibration approaches, allowing the calibration without higher accuracy reference instruments. They can mainly be subdivided in three groups based on the used objects, namely, on the plane-based (Gielsdorf et al., 2004), cylinder-based (Chan and Lichti, 2012) and target-based approaches. The latter one can be furtherly subdivided according to different implementations of the calibration algorithm on the network method (Lichti, 2007), two-face method (Medić et al., 2017; Wang et al., 2017) and length consistency method (Wang et al., 2017). Additionally, there is the highly specific case of the paraboloid-based calibration (Holst and Kuhlmann, 2014).
For a successful calibration, all mentioned approaches require man-made objects that can be well approximated with the geometrical primitives and which are well distributed in the TLSs field of view (FoV). Namely, for the plane- and cylinderbased approaches to work, we need at least several planar or cylindrically shaped objects distributed in the surrounding, such as walls, pipes or street lamps. The target-based approach requires that a certain number of targets (typically planar targets with a certain pattern or spheres) are manually distributed around the measurement area. To perform the TLS selfcalibration in each case we need to solve the problem of the point correspondences between two or more consecutive scans from one or more scanner stations. In the previous approaches, this problem is solved by employing some a priori knowledge about the environment (e.g. we know that some measurements from scan 1 and scan 2 belong to the same wall, target or cylinder).

Our proposed approach differs from the latter ones due to the fact that it does not rely on any geometrically describable manmade objects, which are a priori identified in the surrounding. This makes it an especially interesting solution for the tasks outside the urban areas such as deformation monitoring of landslides and slopes. The correspondent points are found automatically in the scene using the feature matching of $2 \mathrm{D}$ local image keypoints, which are usually used for camera calibration (Liu and Hubbold, 2006) and visual odometry (Nister et al., 2004) for years. Exploiting a nearly continuous spherical scan pattern to represent the 3D TLS measurements with 2D images is already successfully used for the TLS point cloud registration (Urban and Weinmann, 2015). Herein, we use the corresponding keypoints of the image representations for the intensity measurements for the TLS self-calibration.

\footnotetext{
Corresponding author
} 
In order to find multiple keypoint correspondences with sufficient localization accuracy, it is necessary to avoid strong changes in the measurement configuration. Namely, they cause multiple image distortions including the changes in the intensity values. Therefore, we limited our calibration approach on estimating only two-face sensitive CPs, which can be estimated from a single scanner station. We build upon the two-face calibration method by substituting targets with the keypoints. The two-face measurements represent the measurement principle in which the scanner makes two consecutive full panoramic scans from a single station making a full circle around its standing axis. During the $1^{\text {st }}$ scan the instrument rotates for a half of a circle $\left(0^{\circ}-180^{\circ}\right)$, while during the $2^{\text {nd }}$ scan the instrument continues the rotation closing the circle $\left(180^{\circ}\right.$ $360^{\circ}$ ). Such a measurement concept can reveal multiple systematic errors and it is widely used in the geodetic community for the calibration of total stations (Schofield and Breach, 2007), which have a similar construction as TLSs. This assures minimum distortion of the intensity images between consecutive scans. Although, this means that the calibration is not comprehensive, it still allows estimation of the majority of the relevant CPs.

The aim of this article is to introduce an alternative in-situ selfcalibration approach for the panoramic TLSs and compare it with an established calibration approach (in this instance, the target-based two-face calibration method). In order to validate it, we analyzed the parameter correspondence between the approaches, as well as the achieved parameter precisions and correlations on a real dataset. In the end, we analyze the impact of the estimated CPs on the point cloud quality.

The state-of-the-art on the topic, the problem statement and the aim of the article are given in this section. The implemented extraction of 2D keypoints in the context of TLSs and the twoface calibration algorithm, which is a foundation both for the target-based and 2D keypoint-based calibration, are given in Section 2. The experiment conducted to validate the proposed approach is described in Section 3. Section 4 contains the results of the conducted experiment and the following discussion, while the main conclusions are drawn in Section 5.

\section{METHODS}

\subsection{The implemented 2D keypoint extraction}

The panoramic TLS mainly comprises two angular encoders measuring the angular steps, a spinning mirror deflecting the laser beam and an electronic distance measurement (EDM) device, which also records the intensity of the returned signal. The mirror spins around the horizontal (tilt) axis and deflects the laser beam within one vertical profile $\left(0^{\circ}-360^{\circ}\right)$, while the whole system is rotating around the vertical (standing) axis $\left(0^{\circ}-\right.$ $180^{\circ}$ or $180^{\circ}-360^{\circ}$ ). Hence, the whole surrounding is sampled nearly continuously through dense pointwise measurements recording horizontal angles, vertical angles and distances (from further on ranges). These 3D spherical coordinates are internally transformed and stored as Cartesian coordinates (Schofield and Breach, 2007).

To create the image representation of the observed environment, the point coordinates are transformed back to the spherical coordinates Eq. 1:

$$
\begin{aligned}
& r_{j}^{i}=\sqrt{x_{j}^{i^{2}}+y_{j}^{i^{2}}+z_{j}^{i^{2}}}, \\
& \varphi_{j}^{i}=\arctan \left(\frac{x_{j}^{i}}{y_{j}^{i}}\right), \\
& \theta_{j}^{i}=\arccos \left(\frac{z_{j}^{i}}{r_{j}^{i}}\right) .
\end{aligned}
$$

Where $r_{j}^{i}, \varphi_{j}^{i}, \theta_{j}^{i}$ denote the range, horizontal and vertical angle measurements and $x_{j}^{i}, y_{j}^{i}, z_{j}^{i}$ are 3D Cartesian coordinates of the $\mathrm{i}^{\text {th }}$ point measured from the $\mathrm{j}^{\text {th }}$ scanner station. The horizontal angles are further corrected for each quadrant by adding $180^{\circ}$ or $360^{\circ}$ where necessary, and the vertical angles are corrected by subtracting the calculated value from $360^{\circ}$ where necessary. This way, both the horizontal and the vertical circular motions of the instrument can be described with values from $0^{\circ}$ to $360^{\circ}$.

Further, the rasterized grids of the horizontal and vertical angular values are created. Therefore, the grid size corresponds to the angular sweep of one scan, which is $180^{\circ}$ in horizontal and slightly less than $360^{\circ}$ in vertical direction (due to the measurement gap underneath the instrument). The grid resolution corresponds to the selected scanning resolution. Both depend on the instrument properties and can be found in manufacturer's specifications. Finally, the 2D natural nearest neighbor interpolation is used both on the range and intensity values to assign a value for each grid cell based on the recorded data. This interpolation step is necessary because partial measurement gaps are unavoidable in the terrestrial laser scanning. This way, two image representations of the environment are created: the range image and the intensity image. The intensity image is used for the detection of the keypoints, while the distance image is used to calculate back the 3D coordinates of the keypoints. Therefore, the intensity image is transformed from arbitrary (manufacturer dependent) intensity values to an 8-bit grayscale image, in order to allow an efficient image processing, while the range image is preserved in the original state to maintain the high precision.

The latter processes are computationally demanding and a typical TLS point cloud comprises hundreds of millions of points. To tackle this, we cannot down-sample the point cloud due to significant losses in the precision. Hence, the original point cloud is segmented in sections, in two steps. Firstly, eight boundary boxes are defined dissecting the point cloud on four quadrants above the horizontal plane and four quadrants below (Fig.1). Secondly, after obtaining the spherical coordinates of each point cloud segment, the measurements are organized in the ascending order of the horizontal angles and furtherly dissected on the images with the width of $10^{\circ}$. This results in 72 separate segments representing the whole panoramic image of one scan. The same process is applied on both scans of two-face measurements. By the end of this process, there are 72 segment pairs showing nearly identical images, one belonging to the $1^{\text {st }}$, and one to the $2^{\text {nd }}$ scan of the two-face measurements (Fig. 2).

From multiple methods for the detection of image keypoints (Schmid et al., 2000), the Förstner interest point detector (Förstner and Gülch, 1987), which is a form of an interest operator, is selected. It is applied on each of the segment (i.e. image) pairs. This interest operator detects and localizes salient local image features (junctions and circular points) or keypoints, which are distinctive in their neighborhood and are reproduced in corresponding images in a similar way. The 
Förstner operator is selected due to its verified high (sub-pixel) localization accuracy (Rodehorst and Koschan, 2006), which is necessary for the successful instrument calibration. Namely, the achievable TLS point cloud registration accuracy with other features was proven to be in order of centimeters (Urban and Weinmann, 2015), while for the calibration purposes it is necessary to detect sub-millimeter signals. The selected parameter settings are given in appendix A.

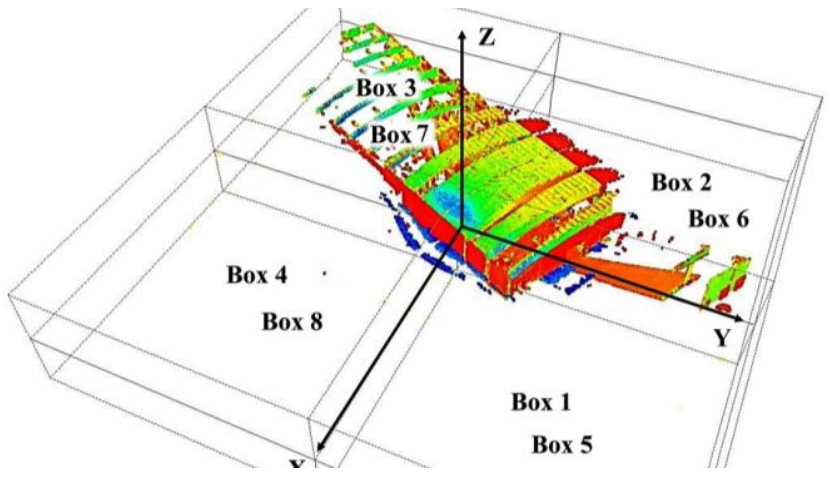

Figure 1 . The point cloud separation by boundary boxes

The localized keypoints need to be extracted and uniquely described with an appropriate feature descriptor in order to allow correct correspondent point search and feature matching between corresponding images. We tested several point descriptors, namely BRISK, SURF, FREAK, KAZE (Hassaballah and Hosny, 2018, p. 116-126), and we selected the BRISK descriptor (Leutenegger et al., 2011), as it provided the highest number of the correct matches in a test run (Fig 2.). However, it should be noted that the differences between descriptors were not large. All of them returned similar number of overall matches ( $2 \%$ differences) and correct matches (71-78 $\%)$. After the feature matching step, the 3D spherical coordinates of the matched features are calculated using the $2 \mathrm{D}$ image coordinates and a linear interpolation on the horizontal and vertical angular grids, as well as the range image. The described process is iteratively repeated for each of the segment pairs.

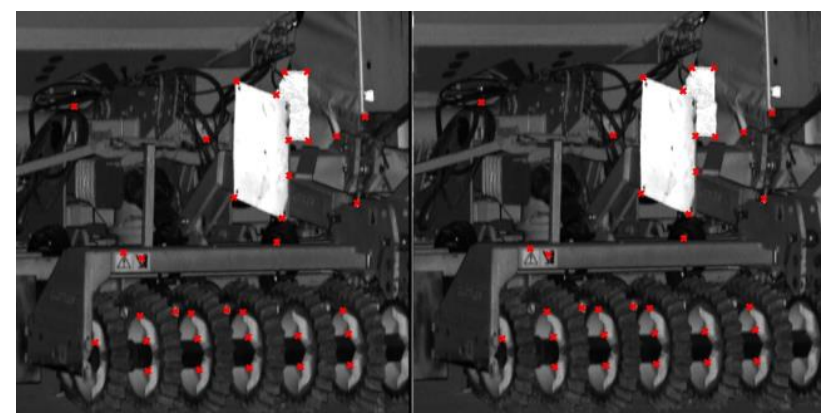

Figure 2. Fractions of the intensity images of 1 segment pair belonging to the $1^{\text {st }}$ scan (left) and the $2^{\text {nd }}$ scan (right) of the two-face measurements (red crosses - matched keypoints).

Additionally, two outlier removal strategies are implemented to cope with the falsely matched keypoints. The first strategy is to test the median absolute deviation (MAD) of the horizontal and vertical differences in $2 \mathrm{D}$ image coordinates between the matched keypoints, for each point cloud segment pair separately. Namely, a notable distortion of the internal image geometry between two consecutive scans of the two-face measurements is not expected. Therefore, all differences should be similar. The second strategy is employed after all point cloud segment pairs are processed. We expect that the only difference between the 3D coordinates of the matched keypoints between the $1^{\text {st }}$ and the $2^{\text {nd }}$ scan of the two-face measurements are due to the TLS misalignments, measurement noise and sampling uncertainty, where all of them have low and predictable magnitudes. Therefore, differences between the 3D coordinates of corresponding points are computed and all keypoints whose differences breach a subjectively defined thresholds are removed (180" for angles, and $0.1 \mathrm{~m}$ for distances - experience based). The remaining keypoints are used as observations in the two-face TLS self-calibration adjustment.

\subsection{Two-face calibration adjustment}

The two-face calibration adjustment is used in this article both as a core of the proposed 2D keypoint-based calibration and for the attested reference, the target-based calibration. As stated in Medić et al., (2017), there are 11 instrumental misalignments relevant for the high-end panoramic laser scanners (Tab. 1). By high-end, we denote instruments having high quality components including angular encoders with multiple reading heads, which eliminates a considerable amount of overall possible TLS misalignments (Muralikrishnan et al., 2015; Walsh, 2015). Nearly all of the relevant misalignments (9 out of 11) are sensitive to two-face measurements (Tab 1.). In short, such a misalignment will cause a systematic error that changes its sign if the measurement point is observed in the front side (front face) or the back side (back face) of the instrument. This causes coordinate differences of the measured points between two faces revealing double the value of the systematic error (Medić et al., 2017).

\begin{tabular}{|c|c|}
\hline Parameter & Description \\
\hline$x_{1 n}$ & Horizontal beam offset $*$ \\
$x_{1 z}$ & Vertical beam offset $*$ \\
$x_{2}$ & Horizontal axis offset $*$ \\
$x_{3}$ & Mirror offset $*$ \\
$x_{4}$ & Vertical index offset $*$ \\
$x_{5 n}$ & Horizontal beam tilt * \\
$x_{5 z}$ & Vertical beam tilt \\
$x_{6}$ & Mirror tilt * \\
$x_{7}$ & Horizontal axis error (tilt) $*$ \\
$x_{10}$ & Rangefinder offset \\
\hline \multicolumn{2}{|c|}{$*$ Two-face sensitive parameters } \\
\hline
\end{tabular}

Table 1. List of the TLS misalignments relevant for the highend panoramic TLSs.

We implemented the two-face adjustment algorithm as proposed in Medić et al., (2017). The functional model is:

$$
\boldsymbol{f}^{\boldsymbol{i}}=\left[\begin{array}{c}
x_{2}^{i} \\
y_{2}^{i} \\
z_{2}^{i}
\end{array}\right]-\left[\begin{array}{l}
x_{1}^{i} \\
y_{1}^{i} \\
z_{1}^{i}
\end{array}\right]=0
$$

where $\boldsymbol{f}^{\boldsymbol{i}}$ is the triplet of functions for each $\mathrm{i}^{\text {th }}$ point measured in the front face $\left({ }_{1}\right)$ and back face $\left({ }_{2}\right)$ of the instrument (adjustment observations). They are related to the spherical coordinates by: 


$$
\left[\begin{array}{c}
x_{j}^{i} \\
y_{j}^{i} \\
z_{j}^{i}
\end{array}\right]=\left[\begin{array}{c}
\left(r_{j}^{i}+\Delta r_{j}^{i}\right) \sin \left(\theta_{j}^{i}+\Delta \theta_{j}^{i}\right) \sin \left(\varphi_{j}^{i}+\Delta \varphi_{j}^{i}\right) \\
\left(r_{j}^{i}+\Delta r_{j}^{i}\right) \sin \left(\theta_{j}^{i}+\Delta \theta_{j}^{i}\right) \cos \left(\varphi_{j}^{i}+\Delta \varphi_{j}^{i}\right) \\
\left(r_{j}^{i}+\Delta r_{j}^{i}\right) \cos \left(\theta_{j}^{i}+\Delta \theta_{j}^{i}\right)
\end{array}\right]
$$

where $\Delta r_{j}^{i}, \Delta \varphi_{j}^{i}, \Delta \theta_{j}^{i}$ are measurement errors. They can be decomposed on the random noise (adjustment residuals) $v_{r_{j}^{i}}, v_{\varphi_{j}^{i}}, v_{\theta_{j}^{i}}$ and the calibration parameters $x_{1 n}, x_{1 z}, \ldots$ (unknowns):

$$
\begin{aligned}
& \Delta r_{j}^{i}=x_{2} \sin \left(\theta_{j}^{i}\right)+v_{r_{j}^{i},} \\
& \Delta \varphi_{j}^{i}=\frac{x_{1 z}}{r_{j}^{i} \tan \left(\theta_{j}^{i}\right)}+\frac{x_{3}}{r_{j}^{i} \sin \left(\theta_{j}^{i}\right)}+\frac{x_{5 z-7}}{\tan \left(\theta_{j}^{i}\right)}+\frac{2 x_{6}}{\sin \left(\theta_{j}^{i}\right)}+v_{\varphi_{j}^{i}}, \\
& \Delta \theta_{j}^{i}=\frac{x_{1 n+2} \cos \left(\theta_{j}^{i}\right)}{r_{j}^{i}}+x_{4}+x_{5 n} \cos \left(\theta_{j}^{i}\right)+v_{\theta_{j}^{i}} .
\end{aligned}
$$

The initial set of 9 two-face sensitive calibration parameters is modified by combining the parameters that are impossible to separate due to the same functional definition. Hence, in the two-face calibration, we aim at estimating 8 CPs (Eq. 4).

The adjustment is iteratively repeated until the convergence criteria is met (the estimated CPs do not change more than $10^{-8}$ in two consecutive iterations). The original implementation in the form of the least squares estimation (strict Gauss-Helmert model) is transformed to a robust estimator, using the modified Danish approach, as introduced in the TLS calibration in Reshetyuk, (2009), p. 73-84. The robust estimation is necessary due to further expected outliers in the matched keypoints. The initial stochastic model (diagonal covariance matrix of observations) for the target-based calibration is based on the theoretical data from the manufacturer's specifications. On the contrary, the initial guess about the keypoints' precision is estimated empirically using two-face differences of the matched keypoints. Namely, we use MAD (due to the expected presence of outliers) divided by square root of 2 (due to variance propagation). After the adjustment has converged, the global test is carried out. If the test results are positive, the adjustment results are accepted. Otherwise, new measurement weights are calculated using the variance component estimation for each polar measurement separately and the whole procedure is repeated with the new covariance matrix (Förstner and Wrobel, 2016, p. 91-93).

\section{EXPERIMENT}

The instrument under investigation is a Leica ScanStation P20. It is a high-end panoramic TLS with a wide field of view $\left(360^{\circ}\right.$ x $270^{\circ}$ ), measuring range of $120 \mathrm{~m}$ and the accuracy of $1 \mathrm{~mm}$ for EDM measurements and 8" for angular measurements (Leica, 2015). The selected resolution is $1.6 \mathrm{~mm}$ at $10 \mathrm{~m}$ resulting in a grid of angular measurements with a resolution of approximately 33 ". Only a single EDM measurement per point was used to reduce measurement time (i.e. quality level 1).

The calibration experiment was conducted in a large machine hall with dimensions of $75 \times 33 \times 9 \mathrm{~m}$ (Fig 3). For the reference solution based on the target-based TLS calibration, 14 custombuilt targets were well distributed over the scanner's field of view (Fig 4). The targets are made of metal plates with the dimensions of $30 \times 30 \mathrm{~cm}$ and a black-white pattern resembling pizza or cake cuts (Fig. 3). As it was demonstrated in Janßen et al. (2019), such a higher pattern complexity assures higher precision of the target centroid detection.

As we aim at evaluating an in-situ TLS self-calibration approach from a single station, only one set of two-face measurements was conducted. That means that we made only two consecutive full panoramic scans from the location indicated in Fig 4. Hence, the whole measurement procedure lasted approximately 30 minutes. In such a short time period, no notable changes in the environment were expected. Hence, no extensive monitoring of the environmental conditions was conducted. Additionally, the main strength of the two-face calibration is that all of the external systematic effects (e.g. incidence angle and refraction) influence repeated measurements identically, and hence, they do not impact on the difference between the $1^{\text {st }}$ and $2^{\text {nd }}$ scan of two-face measurements (same sign and magnitude). This makes the difference of two-face measurements (Eq. 2) as bias free as possible. The instrument was set up on a standard wooden surveying tripod and tribrach. Tripod stabilization (i.e. spider) was also used, due to the smooth floor surface. Finally, the inbuilt dynamic compensator was turned on to level the instrument with the accuracy of 1.5 ".

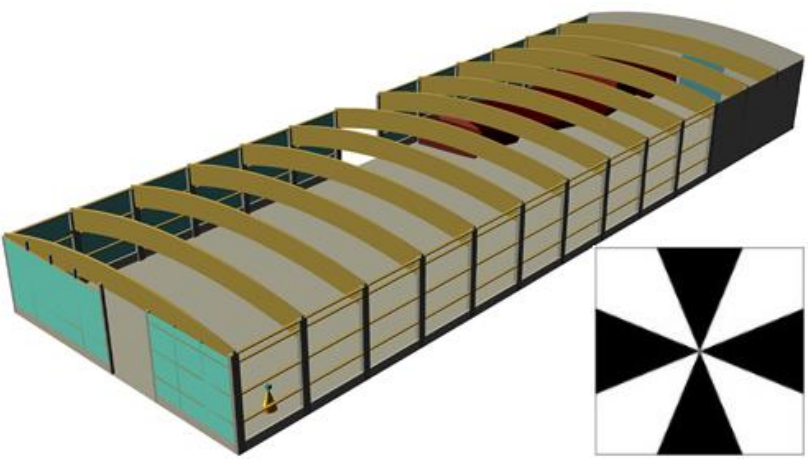

Figure 3. The 3D model of a machine hall and the target template.

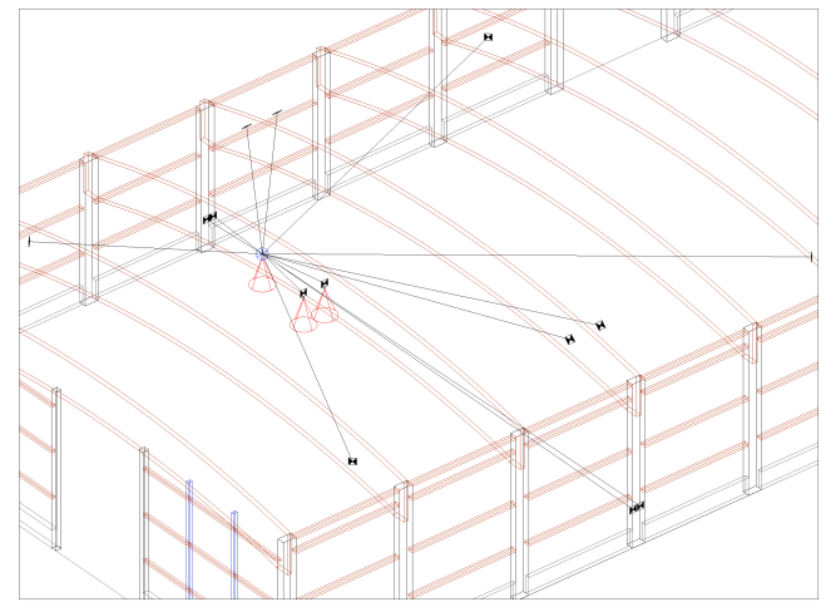

Figure 4. The network configuration of the targets in the machine hall w.r.t. the scanner station with the lines of sight.

The initial data processing was conducted in Leica Cyclone. The point cloud sections belonging to the measured targets were extracted manually. All further computations regarding both the reference and the proposed calibration method were done automatically in MatLab. For the target centroid detection we 
used an algorithm based on the template matching described in Janßen et al. (2019), as it was demonstrated to assure the highest precision of target coordinates. This step provided the observations for the target-based calibration. The observations for the proposed 2D keypoint-based calibration, (keypoints' coordinates) were derived using the approach described in Sec. 2.1. The estimation of the CPs for both calibration methods was done according to Sec. 2.2.

To finally test the impact of the estimated CPs on the overall point cloud quality, the CloudCompare software was used. We computed the differences between the consecutive scans of the two-face measurements before and after applying the calibration parameters. A similar calibration evaluation scheme was used in Holst et al., (2018). In order to increase the sensitivity of this test approach and to reduce the influence of the measurement noise, we used the multiscale model-to-model cloud comparison (M3C2) algorithm (Lague et al., 2013). This algorithm is frequently used in the TLS deformation monitoring tasks to allow the detection of deformations with magnitudes below the measurement noise (Mukupa et al., 2017). All relevant experiment results are presented in the following section.

\section{RESULTS AND DISCUSSION}

\subsection{Measurement residuals}

The reference solution was obtained based on 84 observations with no detected outliers, while the solution of the proposed approach was obtained based on 24978 observations, where $5.9 \%$ of the values were detected as outliers. The distribution of the observed points from a viewpoint of the instrument is presented in Fig 5. It is visible that both the keypoints and the targets are well distributed over the field of view (FoV), assuring an adequate measurement geometry for the successful calibration.

From the initial set of 8 calibration parameters (Eq. 4), one was omitted. Namely, the parameter $\mathrm{x}_{2}$ (horizontal axis offset) influencing the distance measurements could not be successfully estimated by the proposed approach. The reason for this is insufficient precision of the range measurements. If the parameter $x_{2}$ is wrongly estimated (by a large amount) in one of the iterations, it causes exclusion of multiple valid measurements and, consequently, wrong or failed algorithm convergence. The ranges to the feature points are interpolated from individual observations, in contrary to the target-based approach where they are estimated from hundreds or thousands of observations. These differences are visible in Tab. 2, where the measurement standard deviations before and after the calibration are given. The a priori values are calculated as a standard deviation of the two-face differences divided by the square root of 2 , while the a posteriori values are calculated as a standard deviation of the adjustment residuals (both after the outlier removal). The ranges standard deviation of the target centroid is one order of magnitude lower, allowing the successful estimate of the parameter $\mathrm{x}_{2}$. In general, both calibration approaches notably improved the observation accuracy and, hence, proved comparable effectiveness. It is worth noting that the standard deviation improvement (in percentage) for the target-based approach is much higher. This is because the target centroids are much more precise and, therefore, signal (bias due to CPs) to noise ratio is much higher. Hence, when the bias is removed through the calibration, the values decreased drastically.

\begin{tabular}{|c|c|ccc|}
\cline { 3 - 5 } \multicolumn{2}{c|}{} & $\sigma_{r}[\mathrm{~mm}]$ & $\sigma_{\varphi[\text { ["] }}$ & $\sigma_{\theta}["]$ \\
\hline \multirow{2}{*}{ Targets } & a priori & 0.14 & 19.11 & 8.77 \\
& a posteriori & 0.11 & 1.71 & 0.59 \\
\hline 2D & a priori & 1.82 & 8.18 & 7.82 \\
Keypoints & a posteriori & 1.25 & 4.03 & 3.73 \\
\hline
\end{tabular}

Table 2. The standard deviations in ranges, horizontal and vertical angles of the target centroids and 2D keypoints, before and after the calibration.

\subsection{Calibration parameters}

The estimated calibration parameters, their standard deviation and maximal correlations are given in Tab. 3. The parameter values fit closely together between both realizations, but the test results suggest that they are significantly different (congruency, parameter test, one-tailed F-test, 99\% probability). The inspection showed that this difference originates from the parameter $\mathrm{x}_{4}$ (vertical index error). The probable reason lies in the fact that any existing correlations between the highly abundant keypoints are disregarded in the stochastic model due to the insufficient knowledge. This is a common problem in TLS applications and, hence, the statistical test results should be considered with caution (Jurek et al., 2017). Namely, disregarding the observation correlations leads to overestimated accuracy of the calibration parameters. Hence, the threshold for the significant parameter difference is unrealistic (too strict).

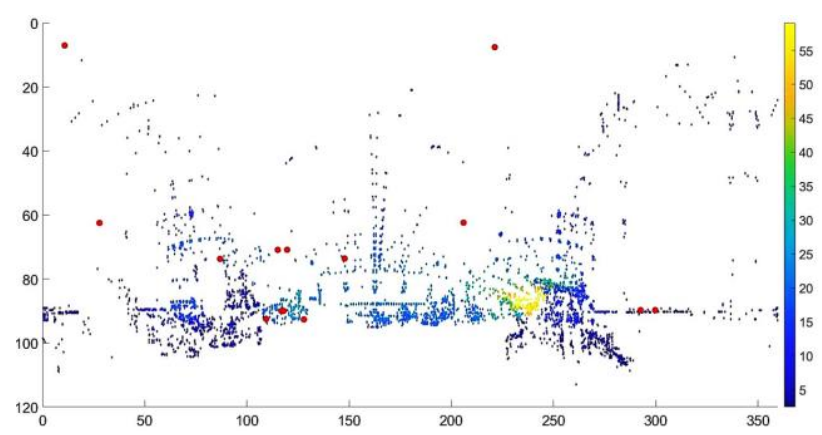

Figure 5. Distribution of the keypoints (multicolored) and targets (red) in the scanner's FoV (x/y axes - horizontal/vertical angles in degrees, color bar - point to scanner distance).

For the proposed approach, all CPs seem to be significant (onetailed Student's t-test, 99\% significance) and estimated with high accuracy (superior to the target-based approach). However, again, these values should probably be scaled for some amount due to the disregarded correlations. The correlations between the CPs are nearly identical in both calibration approaches, despite the huge difference in the number of observations and the resulting measurement configuration. These results are relevant for the active research topic of optimizing the network configuration for the target-based calibration approach (Abbas et al., 2014). In Tab. 3, we can see a notable increase in the standard deviations of some highly correlated CPs in the case of the target-based approach (mostly $\mathrm{x}_{5 \mathrm{z}-7}$ and $\mathrm{x}_{1 \mathrm{z}}$ ), where $\mathrm{a}$ relatively low number of observations is used. That means that a small random error in estimating one of these two parameters can be wrongly interpreted as an influence of the other parameter. Hence, this can significantly bias the calibration results. 


\begin{tabular}{|c|c|c|c|c|}
\hline \multicolumn{5}{|c|}{ Target-based } \\
\hline Parameter & $\mathbf{x}$ & $\sigma_{\mathbf{x}}$ & $\rho_{\mathrm{xy}}$ & With \\
\hline $\mathbf{x}_{4}["]$ & -7.31 & 0.27 & -0.69 & $x_{5 n}$ \\
\hline $\mathbf{x}_{5 n}["]$ & -13.91 & 1.64 & -0.95 & $x_{1 n+2}$ \\
\hline $\mathbf{x}_{5 z-7}["]$ & -8.92 & 5.95 & -0.99 & $\mathrm{x}_{1 \mathrm{z}}$ \\
\hline $\mathbf{x}_{6}["]$ & 1.38 & 0.77 & -0.84 & $\mathrm{x}_{3}$ \\
\hline $\mathbf{x}_{3}[\mathbf{m m}]$ & 0.01 & 0.03 & -0.84 & $x_{6}$ \\
\hline $\mathbf{x}_{1 z}[\mathbf{m m}]$ & 0.04 & 0.15 & -0.99 & $\mathrm{X}_{5 \mathrm{z}-7}$ \\
\hline $\mathbf{x}_{1 \mathrm{n}+2}[\mathrm{~mm}]$ & -0.11 & 0.05 & -0.95 & $x_{5 n}$ \\
\hline \multicolumn{5}{|c|}{ 2D Keypoint-based } \\
\hline Parameter & $\mathbf{x}$ & $\sigma_{\mathrm{x}}$ & $\rho_{\mathrm{xy}}$ & With \\
\hline $\mathbf{x}_{4}["]$ & -6.21 & 0.07 & -0.52 & $x_{5 n}$ \\
\hline $\mathbf{x}_{5 n}["]$ & -15.21 & 0.71 & -0.93 & $x_{1 n+2}$ \\
\hline $\mathbf{x}_{5 \mathrm{z}-7}["]$ & -8.40 & 0.72 & -0.97 & $\mathrm{x}_{1 \mathrm{z}}$ \\
\hline $\mathbf{x}_{6}["]$ & 1.56 & 0.06 & -0.80 & $\mathrm{x}_{3}$ \\
\hline $\mathbf{x}_{3}[\mathrm{~mm}]$ & 0.03 & 0.00 & -0.80 & $x_{6}$ \\
\hline $\mathbf{x}_{1 z}[\mathbf{m m}]$ & -0.15 & 0.02 & -0.97 & $x_{5 z-7}$ \\
\hline $\mathbf{x}_{1 \mathrm{n}+2}[\mathrm{~mm}]$ & -0.10 & 0.03 & -0.93 & $x_{5 n}$ \\
\hline
\end{tabular}

Table 3. The estimated calibration parameters, their standard deviations and maximal mutual correlations for the target-based and the 2D keypoint-based approach.

However, in the case of the keypoint-based approach, where the number of observations is much higher, the standard deviations of these parameters are much lower. This means that high observations' abundance has a positive impact on reducing the possible parameter bias, despite persistently high correlations between functionally similar CPs $\left(\mathrm{x}_{5 \mathrm{z}-7}\right.$ and $\mathrm{x}_{1 \mathrm{z}}, \mathrm{Eq}$. 4). This is rather expected due to the law of large numbers: the higher the number of events (observation errors) is, the lower is the probability that the mean error will fall far away from a zero value. Hence, there is a lower probability to wrongly interpret random errors as an influence of the correlated parameters.

To conclude, at least for some functionally similar CPs, it is hard to achieve a meaningful network configuration that would reduce high correlations. To combat low accuracy due to high correlations, it is necessary to retain a high number of observations. Fortunately, these parameters are two-face sensitive. Hence, the optimal solution for the comprehensive TLS calibration could be a combination of an intelligent target network for the parameters not estimable through the 2D keypoint-based calibration and the vastly redundant 2D keypoints observations for highly correlated parameters.

\subsection{Influence on the point cloud}

In the end, we applied CPs obtained from the 2D keypointbased calibration approach on complete point clouds. We computed the differences between the $1^{\text {st }}$ and $2^{\text {nd }}$ scan of the two-face measurements, before and after the calibration, using the mentioned $\mathrm{M} 3 \mathrm{C} 2$ comparison algorithm. The differences are computed in the direction of the local surface normals. We present the part of the point cloud where the influence of the TLS misalignments is the most prominent (the hall's roof) in Fig 6. The differences are colored based on the offset direction (sign). The zero differences are represented with a green color, while all differences breaching a threshold of $1 \mathrm{~mm}$ are colored blue and red (the values in between vary gradually). The red rhombus denotes the scanner station.
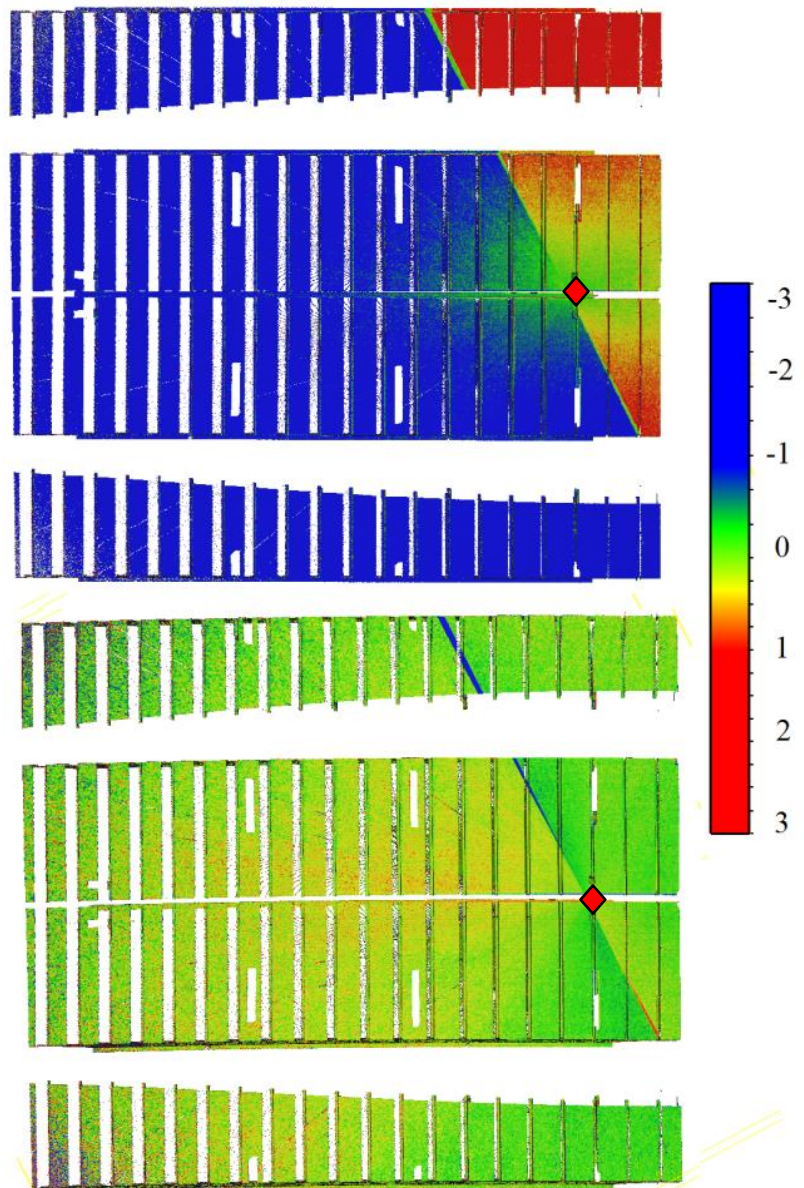

Figure 6. The M3C2 differences between the $1^{\text {st }}$ and the $2^{\text {nd }}$ scan of the roof segment, before (top) and after (bottom) the calibration (in $\mathrm{mm}$ ). Red rhombus denotes the scanner station location.

On the upper part of the figure, we can observe a high systematic trend in the computed differences with an abrupt change in sign between parts that are measured in the front and in the back of the TLS. If it would remain unrevealed, such a systematic influence could be mistaken for roof damage in a deformation analysis. On the lower part of the figure it is visible that applying CPs notably reduced the differences and almost completely removed the systematic effects. The remaining systematic trend can be found in the zone where the start and the end of one panoramic scan are overlapping (a blue-read line). In this zone the points are measured both in the front and in the back of the instrument and these measurements are blended. Hence, it is impossible to correctly convert Cartesian to polar coordinates and hence to apply the CPs correctly. Other than that, the only differences higher than $1 \mathrm{~mm}$ are showing random distribution and they are visible on the far left, away from the scanner station. This increased noise is due to high incidence angles and higher distances, which impacts the quality of the reflectorless distance measurements (Soudarissanane et al., 2011). The associated histograms are presented in Fig. 7. It is visible that the differences before the 
calibration spawned up to $2.5 \mathrm{~mm}$, while they are limited on approximately $0.75 \mathrm{~mm}$ after the calibration. The standard deviation has decreased nearly 3 times from $1.1 \mathrm{~mm}$ to $0.4 \mathrm{~mm}$ and the systematic trend is completely removed. It is worth noting that these values refer to the differences between two point clouds and they contain doubled values of the eventual systematic errors, as well as the noise (reduced) of both scans. Hence, these values demonstrate the achievable accuracy of the point cloud comparison, rather than the quality of individual scans (which is even higher).

\subsection{General discussion about 2D keypoint-based approach}

The proposed in-situ calibration approach successfully mitigated the majority of the angular systematic deviations due to the instrument misalignments (Eq. 4). There is only one remaining misalignment impacting the angular measurements in the high-end panoramic TLSs. This is the vertical beam tilt $\left(\mathrm{x}_{5 \mathrm{z}}\right.$, Tab. 1) which impacts the measured vertical angles and, due to its functional definition, it is not detectable in the difference of two-face measurements. For estimating this $\mathrm{CP}$ it is necessary to make observations from two or more scanner stations with an appropriate measurement configuration, either a priori or insitu. This is beyond the possibilities of the 2D keypoint-based calibration, because it is impossible to a priori define the configuration of the automatically generated keypoints and because, the current state-of-the-art cannot deliver sufficient localization accuracy of matched keypoints for such strong distortions of images between remote viewpoints. Hence, the combination of the proposed approach and the target-based network approach with intelligently reduced number of targets would be the best solution in terms of: a comprehensive calibration, accurate (precise and unbiased) parameter estimates, and minimal effort.

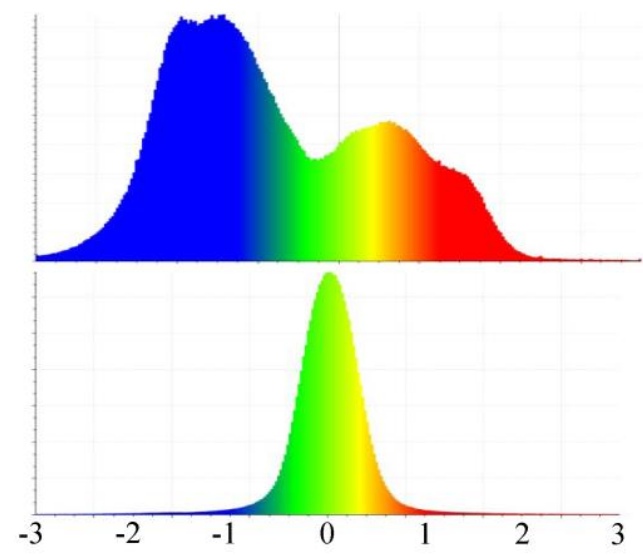

Figure 7. Histograms of the M3C2 differences between the $1^{\text {st }}$ and the $2^{\text {nd }}$ scan of the roof segment, before and after the calibration (in $\mathrm{mm}$ ).

Finally, the main disadvantage of the proposed calibration approach is its inability to compensate for the systematic errors in the range measurements, because they are not two-face sensitive. Many of these systematic errors are successfully mitigated by the manufacturer's calibration and internal instrument corrections, such as rangefinder offset, scale error, cyclic error and intensity related offset. However, again there is a question of the temporal stability of these CPs and considerable research efforts are placed in this direction (Chan et al., 2013; Reshetyuk, 2009, p. 34-42). But definite answers are still missing. Additionally, there is the horizontal axis offset parameter $\left(\mathrm{x}_{2}\right)$ influencing range measurements, which we were unable to estimate, although it is a two-face sensitive parameter. For this it is necessary to improve the precision of the keypoints in the range direction. If the up-to-date angular CPs estimated herein would be supplemented with the up-to-date range CPs, this would lead us on a verge of achieving submillimeter measurement accuracy with high-end TLSs.

\section{CONCLUSION}

This paper has demonstrated a new automatic in-situ selfcalibration method for panoramic TLSs using 2D keypoints. The approach was tested on a dataset with normal working conditions (non-laboratory). The results were compared with the affirmed target-based calibration approach (parameter values, precision and correlations). Finally, the impact of the estimated calibration parameters on the point cloud quality was investigated.

It can be concluded that the proposed in-situ calibration approach successfully mitigated majority of the relevant TLS misalignments, without the need for any preparatory works. This makes it an interesting alternative for the usually employed in-situ approaches, such as the plane- and the cylinder-based approach, especially in an environment where such geometrical shapes cannot be found in abundance. Moreover, in the discussion, we provided rationale for the fusion of the targetbased and the 2D keypoint-based approach. Namely, this presents a good possibility for a comprehensive calibration with an intelligently reduced network of targets. Finally, we successfully demonstrated that applying the estimated calibration parameters completely removed the detectable systematic trends in the point clouds.

There are a lot of possibilities for the further work on this topic, such as: testing different feature detectors, descriptors and their combinations, using additionally or alternatively range images for the feature detection, combining this approach with existing TLS calibration approaches, expanding it on the calibration from multiple stations and improving the keypoint precision in the range direction. Additionally, there is a question of high correlations between several parameters that should be addressed. These steps are planned for the future investigations and promising results may be expected.

\section{REFERENCES}

Abbas, M.A., Lichti, D.D., Chong, A.K., Setan, H., Majid, Z., 2014. An on-site approach for the self-calibration of terrestrial laser scanner. Meas. J. Int. Meas. Confed. 52, $111-123$.

Bianculli, D., Humphries, D., Berkeley, L., 2016. Application of terrestrial laser scanner in particle accelerator and reverse engineering solutions, in: 14th International Workshop Accelerator Alignment. Grenoble, France.

Chan, T.O., Lichti, D.D., 2012. Cylinder-Based Self-Calibration of a Panoramic Terrestrial Laser Scanner. Int. Arch. Photogramm. Remote Sens. Spat. Inf. Sci 39: 169-174.

Chan, T.O., Lichti, D.D., Belton, D., 2013. Temporal Analysis and Automatic Calibration for the Velodyne HDL-32E 
LiDAR System. ISPRS Ann. Photogramm. Remote Sens. Spat. Inf. Sci, 2, pp.61-66.

Förstner, W., Gülch, E., 1987. A Fast Operator for Detection and Precise Location of Distict Point, Corners and Centres of Circular Features, in: Proceedings of the ISPRS Conference on Fast Processing of Photogrammetric Data. Interlaken, pp. 281-305.

Förstner, W., Wrobel, B.P., 2016. Photogrammetric Computer Vision. Springer International Publishing Switzerland.

Gielsdorf, F., Rietdorf, A., Gruendig, L., 2004. A Concept for the Calibration of Terrestrial Laser Scanners. Proc. FIG Work. Week. Athens, Greece 1-10.

Hassaballah, M., Hosny, K.M., 2018. Recent Advances in Computer Vision: Theories and Applications, 1st ed. Springer.

Holst, C., Kuhlmann, H., 2014. Aiming at self-calibration of terrestrial laser scanners using only one single object and one single scan. J. Appl. Geod. 8, 295-310.

Holst, C., Medić, T., Kuhlmann, H., 2018. Dealing with systematic laser scanner errors due to misalignment at area-based deformation analyses. J. Appl. Geod. 12, 169185.

Janßen, J., Medić, T., Kuhlmann, H., Holst, C., 2019. Decreasing the uncertainty of the target centre estimation at terrestrial laser scanning by choosing the best algorithm and by improving the target design. Remote Sens. (under review).

Jurek, T., Kuhlmann, H., Holst, C., 2017. Impact of spatial correlations on the surface estimation based on terrestrial laser scanning. J. Appl. Geod. 11, 143-155.

Lague, D., Brodu, N., Leroux, J., 2013. Accurate 3D comparison of complex topography with terrestrial laser scanner: Application to the Rangitikei canyon (N-Z). ISPRS J. Photogramm. Remote Sens. 82, 10-26.

Leica, 2015. Leica ScanStation P20 Industry's Best Performing Ultra-High Speed Scanner. Leica Scanstation P20 Datasheet.

Leutenegger, S., Margarita, C., Roland, Y.S., 2011. BRISK: Binary robust invariant scalable keypoints, in: Computer Vision (ICCV), 2011 IEEE International Conference On. IEEE. pp. 2548-2555.

Lichti, D.D., 2007. Error modelling, calibration and analysis of an AM-CW terrestrial laser scanner system. ISPRS J. Photogramm. Remote Sens. 61, 307-324.

Liu, J., Hubbold, R., 2006. Automatic Camera Calibration and Scene Reconstruction with Scale-Invariant Features, in: International Symposium on Visual Computing. Springer, Berlin, Heidelberg, pp. 558-568.

Medić, T., Holst, C., Kuhlmann, H., 2017. Towards System Calibration of Panoramic Laser Scanners from a Single Station. Sensors 17, 1145.
Mukupa, W., Roberts, G.W., Hancock, C.M., Al-Manasir, K., 2017. A review of the use of terrestrial laser scanning application for change detection and deformation monitoring of structures. Surv. Rev. 49, 99-116.

Muralikrishnan, B., Ferrucci, M., Sawyer, D., Gerner, G., Lee, V., Blackburn, C., Phillips, S., Petrov, P., Yakovlev, Y., Astrelin, A., Milligan, S., Palmateer, J., 2015. Volumetric performance evaluation of a laser scanner based on geometric error model. Precis. Eng. 40, 139150 .

Nister, D., Naroditsky, O., Bergen, J., 2004. Visual Odometry, in: Computer Vision and Pattern Recognition, 2004. Proceedings of the 2004 IEEE Computer Society Conference. p. vol. 1, 652-659.

Reshetyuk, Y., 2009. Self-calibration and direct georeferencing in terrestrial laser scanning. KTH Stockholm.

Rodehorst, V., Koschan, A., 2006. Comparison and Evaluation of Feature Point Detectors, in: 5th International Symposium Turkish-German Joint Geodetic Days.

Schmid, C., Mohr, R., Bauckhage, C., 2000. Evaluation of interest point detectors. Int. J. Cumputer Vis. 23, 45-78.

Schofield, W., Breach, M., 2007. Engineering Surveying, 6th ed. Elsevier, Oxford.

Soudarissanane, S., Lindenbergh, R., Menenti, M., Teunissen, P., 2011. Scanning geometry: Influencing factor on the quality of terrestrial laser scanning points. ISPRS J. Photogramm. Remote Sens. 66, 389-399.

Urban, S., Weinmann, M., 2015. Finding a good feature detector-descriptor combination for the 2D keypointbased registration of TLS point clouds, in: ISPRS Annals of the Photogrammetry, Remote Sensing and Spatial Information Sciences, ISPRS Geospatial Week 2015. La Grande Motte, France, pp. 121-128.

Walsh, G., 2015. Leica ScanStation White Paper HDR for Leica ScanStation P-Series. Leica Geosystems AG.

Wang, L., Muralikrishnan, B., Rachakonda, P., Sawyer, D., 2017. Determining geometric error model parameters of a terrestrial laser scanner through two-face, lengthconsistency, and network methods. Meas. Sci. Technol. 28(6).

\section{APPENDIX A}

\begin{tabular}{|c|c|}
\hline \multicolumn{2}{|c|}{ Selected parameters for the Förstner operator } \\
\hline Standard deviation of image noise & 1 \\
\hline Filter for gradient & Gaussian 2D \\
\hline Integration kernel & Gaussian \\
\hline Size of derivative filter (sigma) & 1.5 \\
\hline Size of integration filter & 5 \\
\hline Threshold for precision of points & 0.2 \\
\hline Threshold for roundness & 0.3 \\
\hline Significance level for point classification & 0.999 \\
\hline
\end{tabular}

